\title{
Existence of Solutions for Sublinear Kirchhoff Problems with Sublinear Growth
}

\author{
Wei Yang $\mathbb{D},{ }^{1,2}$ Zhan Wang, ${ }^{3}$ and Zhuo Yao $\mathbb{D}^{4}$ \\ ${ }^{1}$ School of Insurance, Southwestern University of Finance and Economics, Chengdu, 611130, China \\ ${ }^{2}$ Collaborative Innovation Center of Financial Security, Southwestern University of Finance and Economics, Chengdu, 611130, China \\ ${ }^{3}$ School of Economics, Southwestern University of Finance and Economics, Chengdu, 611130, China \\ ${ }^{4}$ High Temperature Materials and Magnesite Resources Engineering, University of Science and Technology Liaoning, \\ Anshan, Liaoning 114001, China
}

Correspondence should be addressed to Wei Yang; 2909504391@qq.com

Received 27 February 2019; Accepted 7 April 2019; Published 21 April 2019

Academic Editor: Luigi Rodino

Copyright (C) 2019 Wei Yang et al. This is an open access article distributed under the Creative Commons Attribution License, which permits unrestricted use, distribution, and reproduction in any medium, provided the original work is properly cited.

In this paper, we consider the following sublinear Kirchhoff problems $-\left(a+b \int_{\mathbb{R}^{N}}|\nabla u|^{2} d x\right) \Delta u+V(x) u=f(x, u)$, in $\mathbb{R}^{N}$, where $a>0$ and $b \geq 0$ with $N \geq 3$. A new sublinear growth condition is given. When $f(x, u)$ is not odd in $u$ and not integrable in $x$, we obtain the existence of solutions for the above problem.

\section{Introduction and Main Results}

In this paper, we consider the following nonlinear Kirchhoff type problems:

$$
-\left(a+b \int_{\mathbb{R}^{N}}|\nabla u|^{2} d x\right) \Delta u+V(x) u=f(x, u)
$$

$$
\text { in } \mathbb{R}^{N} \text {, }
$$

where $a, b \geq 0, f(x, u) \in C\left(\mathbb{R}^{N} \times \mathbb{R}, \mathbb{R}\right)$. The Kirchhoff type problems with general potentials on a bounded domain are introduced by

$$
\begin{aligned}
-\left(a+b \int_{\Omega}|\nabla u|^{2} d x\right) \Delta u & =f(x, u), \quad \text { in } \Omega \\
u & =0, \quad \text { on } \partial \Omega
\end{aligned}
$$

which is related to the stationary analogue of the Kirchhoff equation

$$
u_{t t}-\left(a+b \int_{\mathbb{R}^{N}}|\nabla u|^{2} d x\right) \Delta u=g(x, u)
$$

With the development of the variational methods in the last decades, many mathematicians tried to use these methods to search for the existence and multiplicity of solutions for differential equations (see [1-30]). After the work of Lions [14], the Kirchhoff problems have been studied by many mathematicians using the functional analysis methods.

When $f(x, t)$ is superlinear but subcritical with respect to $t$, many mathematicians obtained the existence and multiplicity of solutions for problem (1) (see [4, 9, 15-18, 20, 22$24,30])$. But there are only a few papers concerning the sublinear Kirchhoff type problems. In 2013, Ye and Tang [30] obtained the existence of infinitely many solutions for (1) by symmetrical mountain pass theorem with $f(x, t)=$ $\nu \xi(x)|t|^{\nu-2} t$, where $\nu \in(1,2)$ and $\xi \in L^{2 /(2-v)}\left(\mathbb{R}^{N}, \mathbb{R}^{+}\right)$. In [2], Bahrouni obtained the infinitely many solutions for sublinear Kirchhoff equations with sign-changing potentials. In 2014, Duan and Huang [6] obtained the existence and multiplicity of solutions for problem (1) with more general sublinear nonlinearities. In a recent paper [19], Li and Zhong showed the existence of infinitely many solutions for problem (1) with local sublinear nonlinearities. However, in the above papers, the nonlinear term $f(x, t)$ is assumed to belong to some $L^{p}\left(\mathbb{R}^{N}\right)$ with respect to $x$ for some $p>1$. Under a coercive 
condition, Wang and Han considered a class of sublinear nonlinearities for problem (1) and showed the existence of infinitely many solutions when $f(x, t)$ is odd in $t$ which is the following theorem.

Theorem 1 (see [25]). Suppose that the following conditions are satisfied:

$(V) \inf _{x \in \mathbb{R}^{N}} V(x)>0$ and there are constants $\varrho>0$ and $\alpha>N$ such that, for any $b>0, \lim _{|y| \rightarrow+\infty}$ meas $\left(\left\{x \in B_{\varrho}(y)\right.\right.$ : $\left.\left.V(x) /|x|^{\alpha} \leq b\right\}\right)=0$, where meas $(\cdot)$ denotes the Lebesgue measure;

(s1) there exists a constant $\delta>0$ such that $f \in C\left(\mathbb{R}^{N} \times\right.$ $[-\delta, \delta], \mathbb{R})$ and $f(x,-t)=-f(x, t)$ for all $|t| \leq \delta$ and $x \in \mathbb{R}^{N} ;$

(s2) There is a ball $B_{r_{0}}\left(x_{0}\right)$ such that

$$
\lim \inf _{t \rightarrow 0}\left(\inf _{x \in B_{r_{0}}\left(x_{0}\right)} \frac{F(x, t)}{t^{2}}\right)>-\infty
$$

and

$$
\lim \sup _{t \rightarrow 0}\left(\inf _{x \in B_{r_{0}}\left(x_{0}\right)} \frac{F(x, t)}{t^{2}}\right)=+\infty
$$

where $F(x, t)=\int_{0}^{t} f(x, s) d s$;

(s3) There is a constant $\tau>0$ and a function $g \in$ $C\left([-\tau, \tau], \mathbb{R}^{+}\right)$such that $|f(x, t)| \leq g(t)$ for all $|t| \leq \tau$ and $x \in \mathbb{R}^{N}$.

Then Equation (1) possesses a sequence of weak solutions $u_{n}$ in $X \cap L^{\infty}\left(\mathbb{R}^{N}\right)$ such that $u_{n} \longrightarrow 0$ in $L^{\infty}\left(\mathbb{R}^{N}\right)$ as $n \longrightarrow \infty$.

With coercive condition $(V)$, the authors obtained a new compact embedding theorem, stated as follows.

Lemma 2 (see [25]). Suppose that $V$ satisfies conditions $(V)$. Then $E$ is compact embedded into $L^{p}\left(\mathbb{R}^{N}\right)$ for any $p \in\left[1,2^{*}\right)$.

Remark 3. From Lemma 2, for any $p \in\left[1,2^{*}\right)$, there exists a constant $\eta_{p}>0$ such that

$$
\|u\|_{p} \leq \eta_{p}\|u\| \quad \text { for all } u \in E .
$$

A natural question is whether there exists solution for problem (1) if there is no symmetrical condition on $f(x, t)$ and $f(x, t)$ is not integrable in $x$. In this paper, we try to give an existence theorem on this problem. Motivated by the above papers, we consider problem (1) with some new sublinear nonlinearities and, before we state our result, we assume that $\Gamma$ is a continuous function space such that, for any $\delta(s) \in \Gamma$, there exists a constant $l_{0}>0$ such that

(i) $\delta(s)>0$ for all $s>0$; (ii) $\int_{l_{0}}^{l}(1 / s \delta(s)) d s \longrightarrow+\infty$ as $l \longrightarrow+\infty$.

In order to obtain the critical points of the corresponding functional, we consider the following function space in this paper:

E

$$
:=\left\{u \in H^{1}\left(\mathbb{R}^{N}\right): \int_{\mathbb{R}^{N}}\left(|\nabla u|^{2}+V(x) u^{2}\right) d x<\infty\right\}
$$

with the inner product

$$
(u, v)_{E}=\int_{\mathbb{R}^{N}}(\nabla u \cdot \nabla v+V(x) u v) d x
$$

For any $u \in E$, it follows from (6) that

$$
\widetilde{c}=\inf _{u \in E,\|u\|_{2}=1}\|u\|>0 .
$$

In order to show that the infimum can be achieved, consider a minimizing sequence $\left\{u_{m}\right\} \subset E$ such that

$$
\begin{aligned}
\left\|u_{m}\right\|_{2} & =1, \\
\left\|u_{m}\right\| & \longrightarrow \widetilde{c}
\end{aligned}
$$

$$
\text { as } m \longrightarrow \infty \text {. }
$$

It is easy to see that there exists $\tilde{u} \in E$ such that $u_{m} \rightarrow \tilde{u}$. From Brézis-Lieb Lemma and Lemma 2, we obtain $\|\widetilde{u}\|_{2}=1$, $\|\widetilde{u}\|=\widetilde{c}$ and

$$
\|u\|_{2} \leq \frac{1}{\widetilde{c}}\|u\| \quad \text { for all } u \in E .
$$

Then we state our main result.

Theorem 4. Suppose that $V$ and $f$ satisfy $(V)$ and the following conditions:

( $f 1)$ letting $F(x, t)=\int_{0}^{t} f(x, v) d v$, there exist $\delta(s) \in \Gamma$ with $0<1 / \delta(|s|)<2$ and $l_{\infty}>0$ such that

$$
\begin{aligned}
& 0 \leq t f(x, t) \leq\left(2-\frac{1}{\delta(|t|)}\right) F(x, t) \\
& \qquad \text { for all } x \in \mathbb{R}^{N} \text { and }|t| \geq l_{\infty},
\end{aligned}
$$

( $f 2)$ there exists $\widetilde{d}>\left(\widetilde{c}^{2} / 2\right)(a+1), d_{1}>0$, and $\mu \in\left[1,2^{*}\right)$ such that

$$
F(x, t) \geq \tilde{d t^{2}}-d_{1}|t|^{\mu} \quad \forall(x, t) \in \mathbb{R}^{N} \times \mathbb{R} ;
$$

(f3) $\lim _{t \rightarrow 0} f(x, t)=0$ uniformly in $x$;

( $f 4)$ there is $S>0$ such that

$$
\sup _{x \in \mathbb{R}^{N},|t|=l_{\infty}} F(x, t) \leq S .
$$
(1).

Then there exists at least one nontrivial solution for problem

Remark 5. Let

$$
F(x, t)= \begin{cases}\tilde{c}^{2}(a+1) t^{2} & \text { for }|t| \leq 1 \\ P(t) & \text { for } 1 \leq|t| \leq 2 \\ \frac{t^{2}+2}{\ln \left(t^{2}+2\right)} & \text { for }|t| \geq 2\end{cases}
$$

where $P(t) \in C^{1}(\mathbb{R})$ such that $F(x, t)$ is a $C^{1}$ class function. It is easy to see that $F(x, t)$ satisfies $(f 1)-(f 4)$. However, since $\lim _{t \rightarrow 0}\left(F(x, t) / t^{2}\right)=\widetilde{c}^{2}(a+1)$, we see that $F(x, t)$ does not satisfy $(s 2)$ 
Remark 6. Condition $(f 1)$ is introduced by Wang and Xiao in [26] to prove the existence of periodic solutions for a class of subquadratic Hamiltonian systems. As we know, this is the first time to use such condition on the existence of solutions for sublinear Kirchhoff equations.

\section{Preliminaries}

Lemma 7. Suppose that $(f 1)$ holds; then there exists $d_{2}>0$ such that

$$
|F(x, t)| \leq d_{2} Q(|t|) t^{2} \quad \text { for all } x \in \mathbb{R}^{N} \text { and }|t| \geq l_{\infty} \text {, where } Q(|t|)=\exp \left(-\int_{l_{\infty}}^{|t|} \frac{1}{s \delta(s)} d s\right) \text {. }
$$

Proof. For any $x \in \mathbb{R}^{N}$ and $s \geq l_{\infty} /|t|$, let $w(s)=F(x, s t)$. It follows from condition $(f 1)$ that

$$
w^{\prime}(s) \leq \frac{1}{s}\left(2-\frac{1}{\delta(s|t|)}\right) w(s)
$$

for all $s \geq l_{\infty} /|t|$. Set

$$
T(s)=w^{\prime}(s)-\frac{1}{s}\left(2-\frac{1}{\delta(s|t|)}\right) w(s) .
$$

Then $T(s)=0$ yields that

$$
\begin{aligned}
& w(s) \\
& =\left(\int_{l_{\infty} /|t|}^{s} \frac{w(r)}{r^{2} \delta(r|t|)} d r+\frac{w\left(l_{\infty} /|t|\right) t^{2}}{l_{\infty}^{2}}\right) s^{2} Q(s|t|) .
\end{aligned}
$$

For any $s \geq l_{\infty} /|t|$, (17) shows that $T(s) \leq 0$, which implies that

$$
w(s) \leq \frac{w\left(l_{\infty} /|t|\right) t^{2}}{l_{\infty}^{2}} s^{2} Q(s|t|)
$$

Then we can obtain

$$
\begin{aligned}
F(x, t) & =w(1) \leq \frac{w\left(l_{\infty} /|t|\right) t^{2}}{l_{\infty}^{2}} \eta(|t|) \\
& =\frac{F\left(x,(t /|t|) l_{\infty}\right)}{l_{\infty}^{2}} Q(|t|) t^{2} \text { for }|t| \geq l_{\infty} .
\end{aligned}
$$

From (21) and ( $f 4)$, we can obtain our conclusion.

Remark 8. By the definition of $Q$, it follows from the properties of $\eta$ that $Q(|t|) \longrightarrow 0$ as $|t| \longrightarrow+\infty$.

Lemma 9. Suppose that $V$ satisfies conditions $(f 1),(f 3)$, and $(f 4)$; then, for any $\varepsilon>0$, there exists $d_{\varepsilon}>0$ such that

$$
|f(x, t)| \leq\left(\varepsilon+d_{\varepsilon}|t|\right) \quad \text { for all } x \in \mathbb{R}^{N} \text { and } t \in \mathbb{R}
$$

and

$$
|F(x, t)| \leq \frac{1}{2}\left(\varepsilon|t|+d_{\varepsilon} t^{2}\right)
$$

Proof. From Lemma 7, there exists $d_{3}>0$ such that

$$
F(x, t) \leq d_{3} t^{2} \text { for }|t| \geq l_{\infty} \text {. }
$$

For $|t| \geq l_{\infty}$, it follows from $(f 1)$ that

$$
|f(x, t)| \leq 2 \frac{F(x, t)}{|t|} \leq 2 d_{3}|t| .
$$

We can easily obtain (22) from ( f3) and (25). (23) can be deduced from (22) directly.

\section{Proof of Theorem 1}

By standard arguments, we know that the functional $I: E \longrightarrow$ $\mathbb{R}$, defined by

$$
\begin{aligned}
I(u)= & \frac{a}{2} \int_{\mathbb{R}^{N}}|\nabla u|^{2} d x+\frac{b}{4}\left(\int_{\mathbb{R}^{N}}|\nabla u|^{2} d x\right)^{2} \\
& +\frac{1}{2} \int_{\mathbb{R}^{N}} V(x) u^{2} d x-\int_{\mathbb{R}^{N}} F(x, u) d x,
\end{aligned}
$$

is well defined and the critical points of $I$ are the solutions for problem (1).

Lemma 10. Suppose that $(V),(f 1)-(f 4)$ hold; then I satisfies the (PS) condition.

Proof. From Remark 8 and $\left(V_{1}\right)$, there exists $r_{\infty} \geq l_{\infty}$ such that

$$
Q(|t|) \leq \frac{\inf _{x \in \mathbb{R}^{N}} V(x)}{8 d_{2}} \text { for any }|t| \geq r_{\infty} \text {. }
$$

First, we show that $\left\{u_{n}\right\}$ is bounded in $E$. It follows from the definition of $I,(16)$ and (27), that

$$
\begin{aligned}
I\left(u_{n}\right)= & \frac{a}{2} \int_{\mathbb{R}^{N}}\left|\nabla u_{n}\right|^{2} d x+\frac{b}{4}\left(\int_{\mathbb{R}^{N}}\left|\nabla u_{n}\right|^{2} d x\right)^{2} \\
& +\frac{1}{2} \int_{\mathbb{R}^{N}} V(x) u_{n}^{2} d x-\int_{\mathbb{R}^{N}} F\left(x, u_{n}\right) d x \\
\geq & \frac{a}{2} \int_{\mathbb{R}^{N}}\left|\nabla u_{n}\right|^{2} d x+\frac{1}{2} \int_{\mathbb{R}^{N}} V(x) u_{n}^{2} d x
\end{aligned}
$$




$$
\begin{aligned}
& -\int_{\left|u_{n}\right| \geq r_{\infty}} F\left(x, u_{n}\right) d x \\
& -\int_{\left|u_{n}\right| \leq r_{\infty}} F\left(x, u_{n}\right) d x \\
\geq & \frac{a}{2} \int_{\mathbb{R}^{N}}\left|\nabla u_{n}\right|^{2} d x+\frac{1}{2} \int_{\mathbb{R}^{N}} V(x) u_{n}^{2} d x \\
& -d_{2} \int_{\left|u_{n}\right| \geq r_{\infty}} Q\left(\left|u_{n}\right|\right) u_{n}^{2} d x \\
& -\frac{1}{2} \int_{\left|u_{n}\right| \leq r_{\infty}}\left(\varepsilon\left|u_{n}\right|+d_{\varepsilon} u_{n}^{2}\right) d x \\
\geq & \frac{a}{2} \int_{\mathbb{R}^{N}}\left|\nabla u_{n}\right|^{2} d x+\frac{3}{8} \int_{\mathbb{R}^{N}} V(x) u_{n}^{2} d x \\
& -\frac{1}{2}\left(\varepsilon+d_{\varepsilon} r_{\infty}\right) \int_{\left|u_{n}\right| \leq r_{\infty}}\left|u_{n}\right| d x \\
\geq & \min \left\{\frac{a}{2}, \frac{3}{8}\right\}\left\|u_{n}\right\|^{2}-\frac{\eta_{1}}{2}\left(\varepsilon+d_{\varepsilon} r_{\infty}\right)\left\|u_{n}\right\|,
\end{aligned}
$$

which implies that $I$ is bounded from below on $E$. Since we have Lemma 2, it follows from a standard argument, we obtain that $\left\|u_{n}-u_{0}\right\| \longrightarrow 0$ as $n \longrightarrow \infty$. Hence $I$ satisfies the $(P S)$ condition.

Proof of Theorem 1. By above discussions, we can see that $I$ is of $C^{1}$ class and satisfies the (PS) condition. Similar to (28), we obtain that $I$ is bounded from below. Then $c=\inf _{E} I(u)$ is a critical value of $I$ and there exists $\bar{u}$ such that $I(\bar{u})=c$. Finally, we show that $\bar{u} \neq \equiv 0$. With $\theta>0$ being small enough, it follows from $(f 2)$ and (11) that

$$
\begin{aligned}
I(\theta \widetilde{u})= & \frac{a \theta^{2}}{2} \int_{\mathbb{R}^{N}}|\nabla \widetilde{u}|^{2} d x+\frac{b \theta^{4}}{4}\left(\int_{\mathbb{R}^{N}}|\nabla \widetilde{u}|^{2} d x\right)^{2} \\
& +\frac{\theta^{2}}{2} \int_{\mathbb{R}^{N}} V(x) \tilde{u}^{2} d x-\int_{\mathbb{R}^{N}} F(x, \theta \widetilde{u}) d x \\
\leq & \frac{a \theta^{2}}{2} \int_{\mathbb{R}^{N}}|\nabla \widetilde{u}|^{2} d x+\frac{b \theta^{4}}{4}\left(\int_{\mathbb{R}^{N}}|\nabla \widetilde{u}|^{2} d x\right)^{2} \\
& +\frac{\theta^{2}}{2} \int_{\mathbb{R}^{N}} V(x) \tilde{u}^{2} d x-\widetilde{d} \theta^{2} \int_{\mathbb{R}^{N}} \widetilde{u}^{2} d x \\
& +d_{1} \theta^{\mu} \int_{\mathbb{R}^{N}} \widetilde{u}^{\mu} d x \\
\leq & \left(\frac{1}{2} \widetilde{c}^{2}(a+1)-\widetilde{d}\right) \theta^{2} \\
& +\frac{b \theta^{4}}{4}\left(\int_{\mathbb{R}^{N}}|\nabla \tilde{u}|^{2} d x\right)^{2}+d_{1} \theta^{\mu} \eta_{\mu}^{\mu} \widetilde{c}^{\mu}<0 .
\end{aligned}
$$

Then we can deduce $\inf _{E} I(u)<0$, which implies that $\bar{u} \not \equiv$ 0 .

\section{Abbreviations}

(PS) condition: Palais-Smale condition.

\section{Data Availability}

Data sharing is not applicable to this article as no datasets were generated or analysed during the current study.

\section{Conflicts of Interest}

The authors declare that they have no competing interests.

\section{Authors' Contributions}

The research and writing of this manuscript were a collaborative effort from both of the authors. Zhuo Yao and Wei Yang discussed many details of the problems together. Yao managed this manuscript and Yang revised it. Both of the authors read and approved the final version of the manuscript.

\section{Acknowledgments}

The financial support of National Science and Technology Support Plan Projects of China (no. 2014BAB02B03) is gratefully acknowledged.

\section{References}

[1] H. Berestycki and P. Lions, "Nonlinear scalar field equations I. existence of a ground state," Archive for Rational Mechanics and Analysis, vol. 82, no. 4, pp. 313-345, 1983.

[2] A. Bahrouni, "Infinitely many solutions for sublinear Kirchhoff equations in $\mathbb{R}^{N}$ with sign-changing potentials," Electronic Journal of Differential Equations, vol. 2013, article 98, 8 pages, 2013.

[3] T. Bartsch, Z.-Q. Wang, and M. Willem, "The Dirichlet problem for superlinear elliptic equations," in Stationary Partial Differential Equations. Vol. II, Handbook of Differential Equations, pp. 1-55, Elsevier, North-Holland, Amsterdam, 2005.

[4] J. Chen, "Multiple positive solutions to a class of Kirchhoff equation on R3 with indefinite nonlinearity," Nonlinear Analysis. Theory, Methods \& Applications. An International Multidisciplinary Journal, vol. 96, pp. 134-145, 2014.

[5] L. Ding, L. Li, and J.-L. Zhang, "Solutions to Kirchhoff equations with combined nonlinearities," Electronic Journal of Differential Equations, vol. 10, pp. 1-10, 2014.

[6] L. Duan and L. Huang, "Infinitely many solutions for sublinear Schrödinger-Kirchhoff-type equations with general potentials," Results in Mathematics, vol. 66, no. 1-2, pp. 181-197, 2014.

[7] Z. Guo, "Ground states for Kirchhoff equations without compact condition," Journal of Differential Equations, vol. 259, no. 7, pp. 2884-2902, 2015.

[8] T.X. Hu and W. Shuai, "Multi-peak solutions to Kirchhoff equations in $\mathbb{R}^{3}$ with general nonlinearity," Journal of Differential Equations, vol. 265, no. 8, pp. 3587-3617, 2018.

[9] X. M. He and W. M. Zou, "Exitence and concentration behavior of positive solutions for a Kirchhoff eqution in $\mathbb{R}^{3}$," Journal of Differential Equations, vol. 252, no. 2, pp. 1813-1834, 2012.

[10] S. Júnior, R. Joao, and G. Siciliano, "On a generalized Kirchhoff equation with sublinear nonlinearities," Mathematical Methods in the Applied Sciences, vol. 40, no. 10, pp. 3493-3503, 2017.

[11] G. Kirchhoff, Mechanik, Teubner, Leipzig, Germany, 1883. 
[12] S.-S. Lu, "Multiple solutions for a Kirchhoff-type equation with general nonlinearity," Advances in Nonlinear Analysis, vol. 7, no. 3, pp. 293-306, 2018.

[13] J. Lee, J.-M. Kim, and Y.-H. Kim, "Existence and multiplicity of solutions for Kirchhoff Schrödinger type equations involving $\mathrm{p}(\mathrm{x})$-Laplacian on the entire space $\mathbb{R}^{N}$," Nonlinear Analysis: Real World Applications, vol. 45, pp. 620-649, 2019.

[14] J. L. Lions, "On some questions in boundary value problems of mathematical physics," in Proceedings of the International Symposium on Continuum Mechanics and Partial Differential Equations, vol. 30, pp. 284-346, North-Holland Mathematics Studies, North- Holland, Amsterdam, 1987.

[15] Y. Li, F. Li, and J. Shi, "Existence of a positive solution to Kirchhoff type problems without compactness conditions," Journal of Differential Equations, vol. 253, no. 7, pp. 2285-2294, 2012.

[16] W. Liu and X. He, "Multiplicity of high energy solutions for superlinear Kirchhoff equations," Applied Mathematics and Computation, vol. 39, no. 1-2, pp. 473-487, 2012.

[17] Y. Q. Li, Z.-Q. Wang, and J. Zeng, "Ground states of nonlinear Schrödinger equations with potentials," Annales de l'Institut Henri Poincaré (C) Analyse Non Linéaire, vol. 23, no. 6, pp. 829837, 2006.

[18] Q. Q. Li and X. Wu, "A new result on high energy solutions for Schrödinger-Kirchhoff type equations in $\mathbb{R}^{N}$," Applied Mathematics Letters, vol. 30, pp. 24-27, 2014.

[19] L. Li and X. Zhong, "Infinitely many small solutions for the Kirchhoff equation with local sublinear nonlinearities," Journal of Mathematical Analysis and Applications, vol. 435, no. 1, pp. 955-967, 2016.

[20] J. J. Nie, "Existence and multiplicity of nontrivial solutions for a class of Schrödinger-Kirchhoff-type equations," Journal of Mathematical Analysis and Applications, vol. 417, no. 1, pp. 6579, 2014.

[21] D. Sun and Z. Zhang, "Existence and asymptotic behaviour of ground state solutions for Kirchhoff-type equations with vanishing potentials," Zeitschrift für Angewandte Mathematik und Physik. ZAMP. Journal of Applied Mathematics and Physics. Journal de Mathématiques et de Physique Appliquées, vol. 70, no. 37, 2019.

[22] J. Sun, L. Li, M. Cencelj, and B. Gabrovšek, "Infinitely many sign-changing solutions for Kirchhoff type problems in $\mathbb{R}^{3}$," Nonlinear Analysis, 2018.

[23] J. Sun and S. Liu, "Nontrivial solutions of Kirchhoff type problems," Applied Mathematics Letters, vol. 25, no. 3, pp. 500504, 2012.

[24] X. Wu, "Existence of nontrivial solutions and high energy solutions for Schrödinger-Kirchhoff-type equations in $\mathbb{R}^{N}$," Nonlinear Analysis: Real World Applications, vol. 12, no. 2, pp. 12781287, 2011.

[25] L.-L. Wang and Z.-Q. Han, "Multiple small solutions for Kirchhoff equation with local sublinear nonlinearities," Applied Mathematics Letters, vol. 59, pp. 31-37, 2016.

[26] Z. Wang and J. Xiao, "On periodic solutions of subquadratic second order non-autonomous Hamiltonian systems," Applied Mathematics Letters, vol. 40, pp. 72-77, 2015.

[27] D.-L. Wu, C. Li, and P. Yuan, "Multiplicity solutions for a class of fractional Hamiltonian systems with concave-convex potentials," Mediterranean Journal of Mathematics, vol. 15, no. 35, 2018.
[28] D.-L. Wu, C.-L. Tang, and X.-P. Wu, "Homoclinic orbits for a class of second-order Hamiltonian systems with concaveconvex nonlinearities," Electronic Journal of Qualitative Theory of Differential Equations, vol. 6, pp. 1-18, 2018.

[29] L.-P. Xu and H. Chen, "Ground state solutions for Kirchhofftype equations with a general nonlinearity in the critical growth," Advances in Nonlinear Analysis, vol. 7, no. 4, pp. 535546, 2018.

[30] Y. W. Ye and C. L. Tang, "Multiple solutions for Kirchhoff-type equations in $\mathbb{R}^{N}$," Journal of Mathematical Physics, vol. 54, no. 8, Article ID 081508, 2013. 


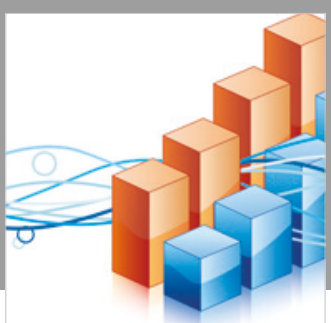

Advances in

Operations Research

\section{-n-m}
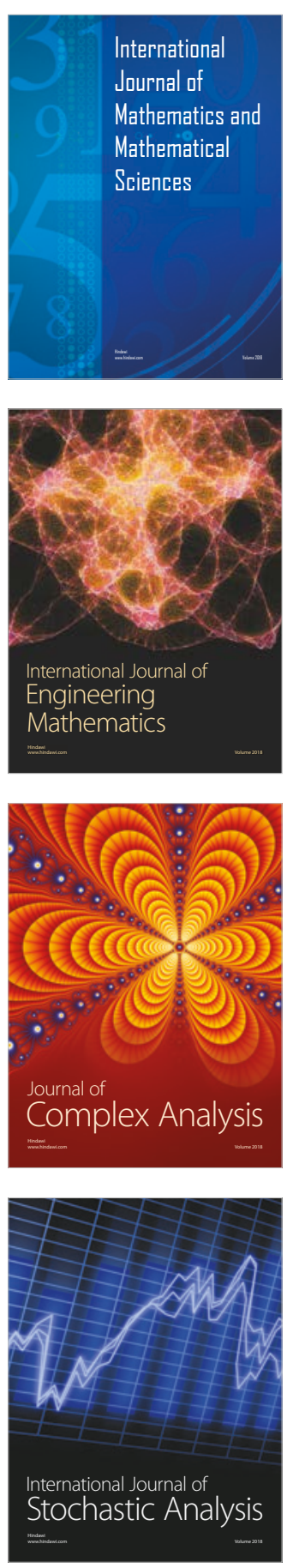
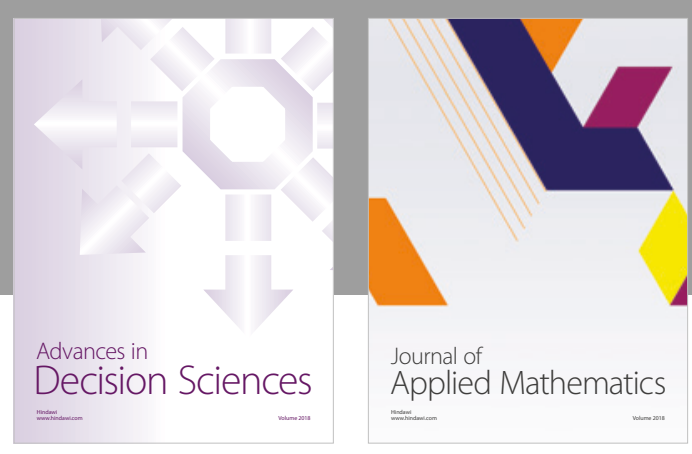

Journal of

Applied Mathematics
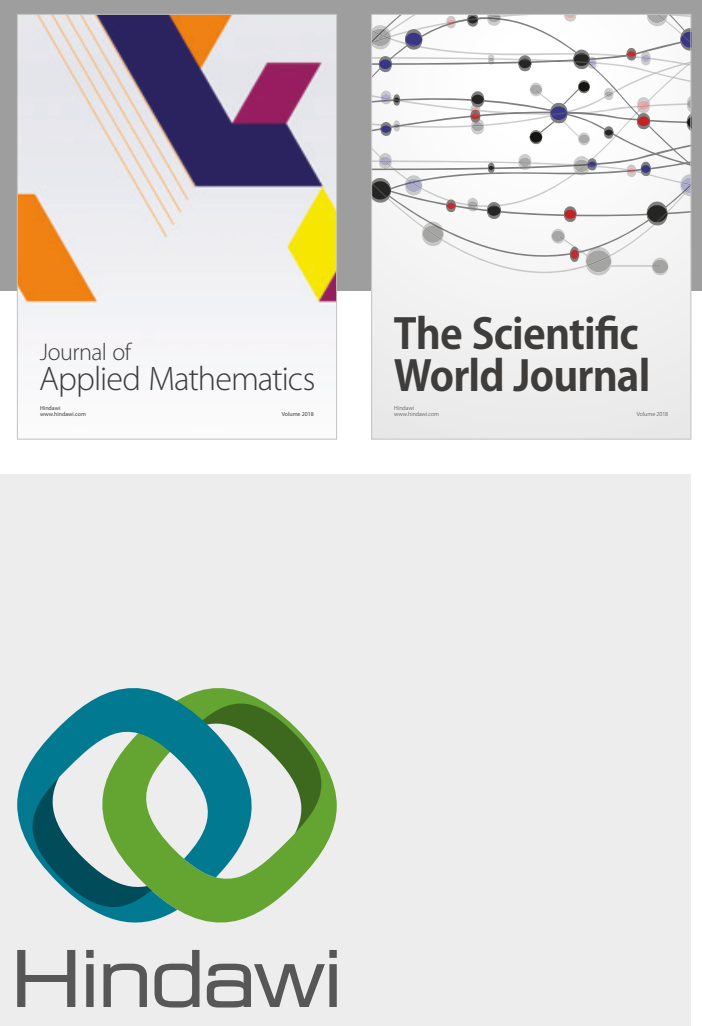

Submit your manuscripts at

www.hindawi.com

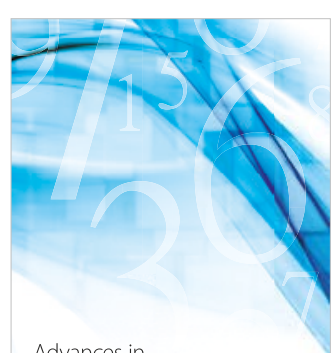

Advances in
Numerical Analysis
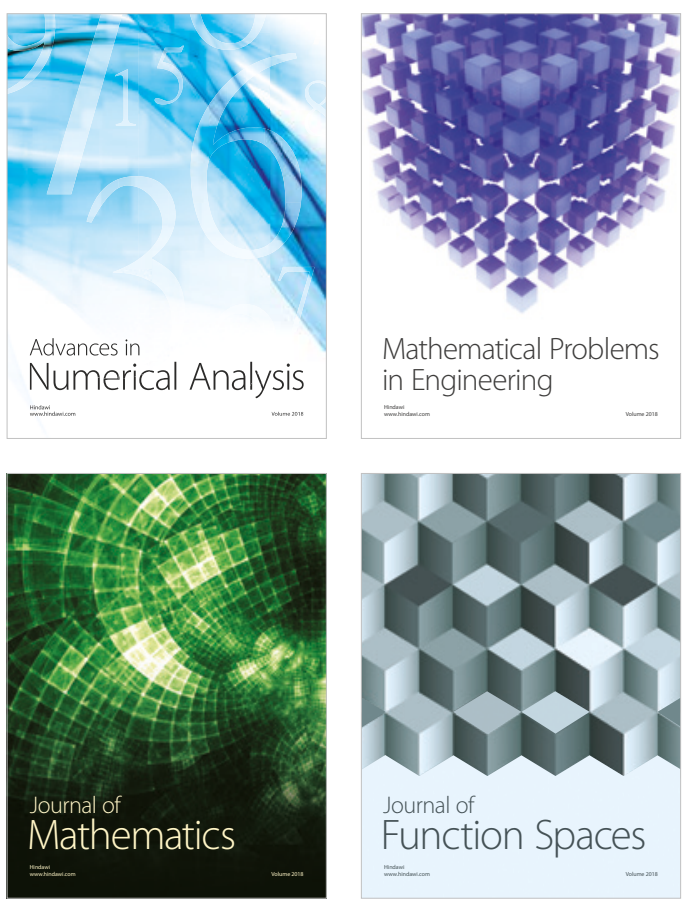

Mathematical Problems in Engineering

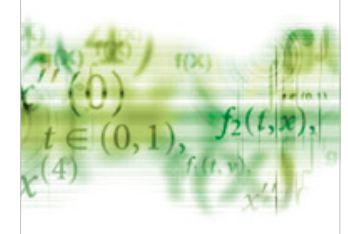

International Journal of

Differential Equations

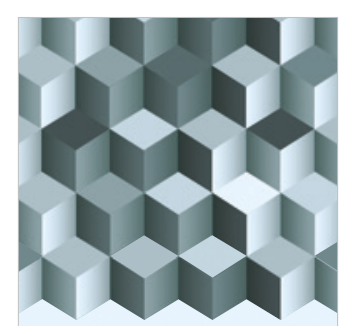

Journal of

Function Spaces

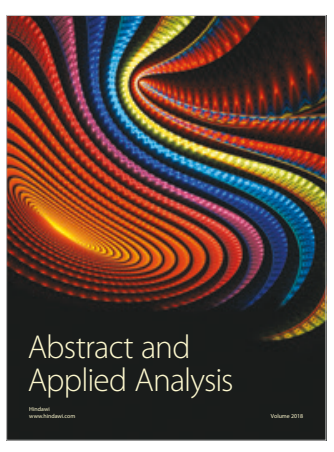

The Scientific

World Journal

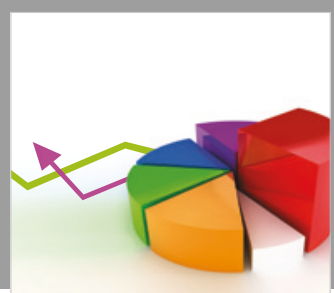

Journal of

Probability and Statistics
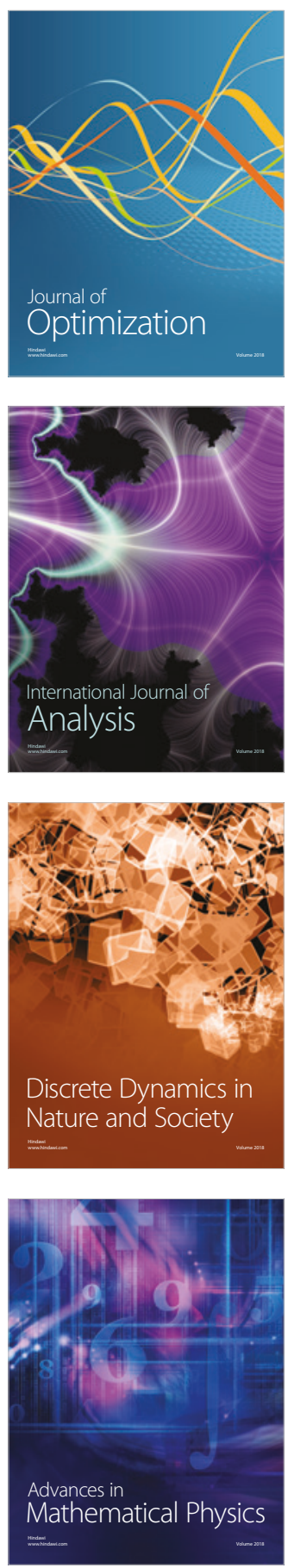